\title{
Effects of number of items and visual display variability on same-different discrimination behavior
}

\author{
LEYRE CASTRO \\ University of Iowa, Iowa City, Iowa \\ MICHAEL E. YOUNG \\ Southern Illinois University, Carbondale, Illinois \\ and \\ EDWARD A. WASSERMAN \\ University of Iowa, Iowa City, Iowa
}

\begin{abstract}
We explored college students' discrimination of complex visual stimuli that involved multiple-item displays. The items in each of the displays could be all the same, all different, or diverse mixtures of some same and some different items. The participants had to learn which of two arbitrary responses was correct for each of the displays without being told about the sameness or differentness of the stimuli. We observed a general improvement in discrimination performance-a rise in choice accuracy and a fall in reaction time-as the number of icons in the display was increased, even when the participants had been trained from the outset with displays containing different numbers of items and when smaller numbers of items were not randomly distributed but grouped in the center of the display. The participants' discrimination behavior also depended on the mixture of same and different items in the displays. Striking individual differences in the participants' discrimination behavior disclosed that people sometimes respond as do pigeons and baboons trained with a similar task. This and previous related research suggest that variability discrimination may lie at the root of same-different categorization behavior.
\end{abstract}

In daily life, people are confronted with many decisions about whether multiple events are the same as or different from one another. We buy a shirt styled differently from those we already own, yet we purchase the same brand of tomato sauce. We order a meal in a restaurant differing from yesterday's breakfast, yet we read the same morning newspaper. Despite the ubiquity of same-different discrimination in adaptive action, the psychological science of such discriminations is still in its infancy (Wasserman, Young, \& Cook, 2004).

Some situations afford easier or harder same-different discriminations. If we were asked whether two or more items are the same as or different from one another, we might imagine that a report of "different" would more readily be prompted by the collection $\mathrm{AB}$ than by the collection ABCDEFGH; after all, less information processing and fewer memory resources should be required when the number of nonidentical items is small than when the number is large. Indeed, people make more errors and respond more

We thank Jessie Peissig for her help in conducting this study. Correspondence concerning this article should be addressed to E. A. Wasserman, Department of Psychology, University of Iowa, Iowa City, IA 52242 (e-mail: ed-wasserman@uiowa.edu). slowly when enumerating large collections of items than when enumerating small collections of items (Kaufman, Lord, Reese, \& Volkmann, 1949; Taves, 1941; Trick \& Pylyshyn, 1994). It therefore seems reasonable to believe that if we were to judge the sameness or differentness of a set of items, this judgment would be easier with a small set than with a large set. But as we will soon see, this reasonable belief may not always accord with the behavioral evidence.

\section{Comparative Psychology of Same-Different Discrimination}

Humans are not alone in having to inspect and judge whether sets of stimuli are the same as or different from one another; nonhuman animals too must be able to discriminate between same and different stimulus sets. Pigeons, for example, might readily take flight with a flock of birds from the same species but be cautious and refrain from taking wing with a flock of birds from different species.

Early efforts to teach pigeons to report whether two visual items - the smallest number that is needed to make a same-different discrimination - are the "same" as or "different" from one another (e.g., Edwards, Jagielo, \& Zentall, 1983; Santiago \& Wright, 1984; Wright, Santiago, Sands, Kendrick, \& Cook, 1985; Wright, Santiago, Urcuioli, \& Sands, 1983) were not especially successful. 
This observation prompted some theorists to conclude that a same-different discrimination requires a relational judgment that is beyond the ken of pigeons and other nonhuman animals (Mackintosh, 2000). But it is also possible, and perhaps even easier, for organisms to make same-different discriminations with sets of more than two items.

Over the past 10 years, research in our Iowa laboratory has been done to investigate the pigeon's discrimination of visual arrays comprising multiple items, usually 16 in number (see Figure 1). When all of those items were identical (same array), we required the pigeons to peck one button to obtain food reinforcement; when all of those items were nonidentical (different array), we required the pigeons to peck a second button to obtain food reinforcement. The pigeons readily learned this discrimination to high levels of accuracy and later transferred the discrimination, with little decrement, to both identical and nonidentical arrays constructed from novel visual items (Wasserman, Hugart, \& Kirkpatrick-Steger, 1995).

But the same and the different displays in Wasserman et al. (1995) also differed in their spatial orderliness; the same displays possessed horizontal and verti- cal regularities that the different displays lacked. The pigeons could be performing a perceptual, rather than a conceptual, discrimination. Nonetheless, when the pigeons were trained to discriminate 16-icon same from 16-icon different arrays in which the items were randomly placed, the birds readily acquired the discrimination and showed excellent transfer to additional arrays (Young \& Wasserman, 1997). Moreover, spatially disarranging the icons or mixing planar rotations in an array did not adversely affect the pigeons' choice responding (Young \& Wasserman, 2001b). And when the pigeons were shown lists of same and different items on a one-at-a-time basis (a successive same-different discrimination; Young, Wasserman, \& Dalrymple, 1997) thereby making spatial orderliness an unusable cue for solving the discrimination - the pigeons again exhibited excellent discriminative performance. These results thus suggest that the pigeons acquired a same-different concept. And because we were more successful than other workers who had presented pigeons with arrays of only two items, these results further suggested that the number of displayed items might be of crucial importance to the birds' discrimination.

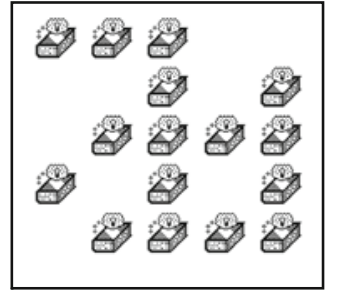

16-icon Same

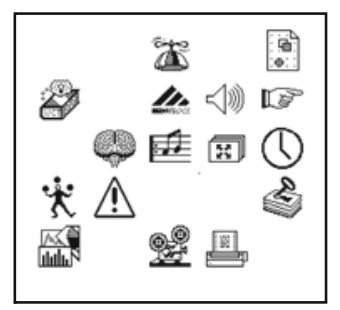

16-icon Different

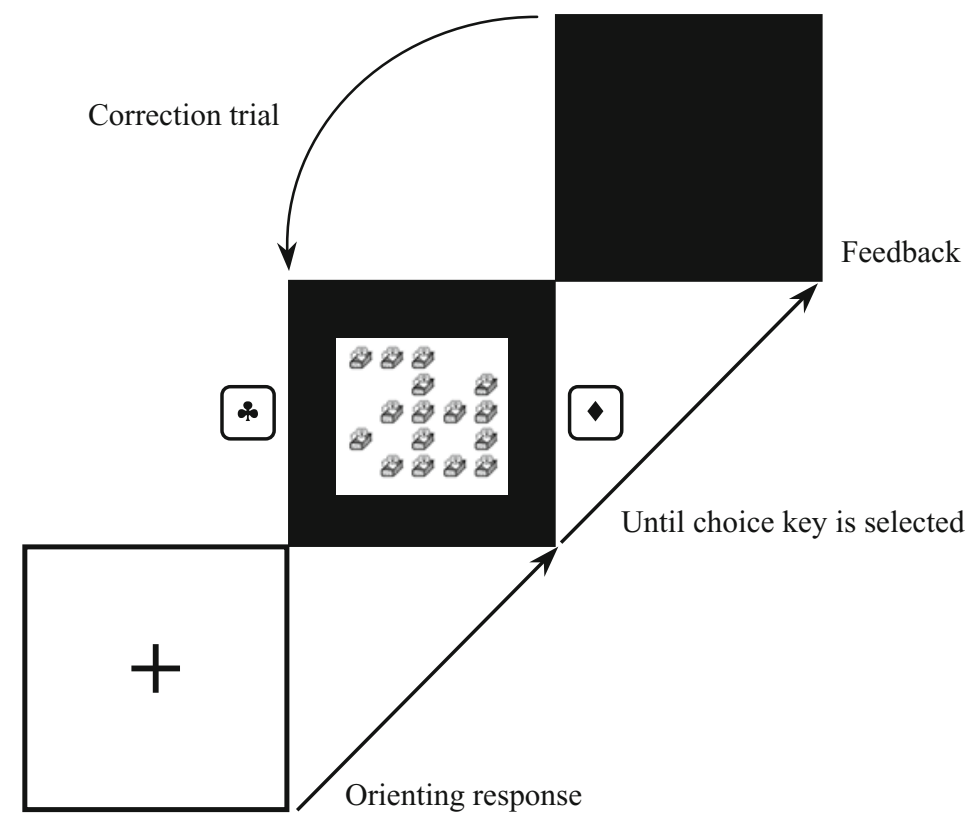

Figure 1. Left panel: Examples of the 16-icon same and different displays used in Young and Wasserman (1997) and subsequent studies. Right panel: Sequence of events in the course of any given trial. The original discrimination task for pigeons was later adapted for the human studies. In both cases, the procedure was very similar. Each trial began with an orienting stimulus, a black cross on a white background, after which the training display appeared at the center of the screen. The pigeons had to peck the display a certain number of times and then select one of the two choice responses that were presented to the left and right of the display. If the choice was correct, the pigeons received food; if the choice was incorrect, the pigeons had to repeat the trial until they made the correct response. In the present experiment, human participants had to press one of two choice keys on the keyboard. A correct choice response was followed by a pleasant tone and the blackening of the display area; an incorrect choice response was followed by a flash of the display area and the repetition of the trial until the correct response was made. 
Before detailing the parametric effects of the number of items on pigeons' discrimination behavior, we investigated the effects of various mixtures of 16-icon displays, in which some icons were the same as one another and other icons were different from one another (see Figure 2). If the pigeons responded categorically, these mixture displays should have prompted reports of different, because they never involved 16 identical items. But when the pigeons were shown arrays containing different mixtures of some identical and some nonidentical items, they responded continuously: As the visual display gradually changed from exhibiting low to high levels of variability, the pigeons' responses progressively changed from "same" to "different"; that is, the pigeons' pecking the "same" or "different" button was a direct function of the variability of the mixture array (Young \& Wasserman, 1997, 2002; Young, Wasserman, \& Garner, 1997). This finding points to display variability as the controlling dimension in the pigeon's discrimination of same from different displays.

Variability in a continuous variable is often quantified using variance. A 16-icon display, however, represents a frequency distribution of a categorical variable: icon type. For each display, there are 16 possible icon types, each of which may have a frequency ranging from 0 (not present) to 16 (the only type present). When a pigeon responds to an array involving an intermediate degree of variability, it must determine whether the value is more similar to that represented by the same arrays or to that represented by the different arrays. The same arrays entail the lowest possible variability; the different arrays exhibit the highest possible variability. Information theory (Shannon \& Weaver, 1949) offers a single quantitative measure of categorical variability: entropy.

To quantify entropy, one can use the following equation (Shannon \& Weaver, 1949):

$$
H(D)=-\sum_{a \in D} p_{a} \log _{2} p_{a}
$$

where $H(D)$ is the entropy of display $D, a$ is a type of item in $D$, and $p_{a}$ is the proportion of items of that type within the display. When a display has 16 identical icons, there is only one category, with a probability of occurrence of 1.0. The different displays consist of one occurrence of each of 16 icons or categories, yielding an entropy of 4.0. During mixture testing, the pigeons must thus respond to a display based on whether its entropy is closer to 0.0 or to 4.0 , the two endpoints of the entropy dimension.

What about the effect of the number of items in same and different displays? Pigeons' discrimination of same from different visual arrays turned out to be notably weaker when there were fewer items in a display. This decrease in discriminative performance as the number of items was reduced from training (with 16 icons) to testing (with 2, $4,8,12$, and 14 icons) was primarily due to a decline in the correct discrimination of different arrays; the discrimi-

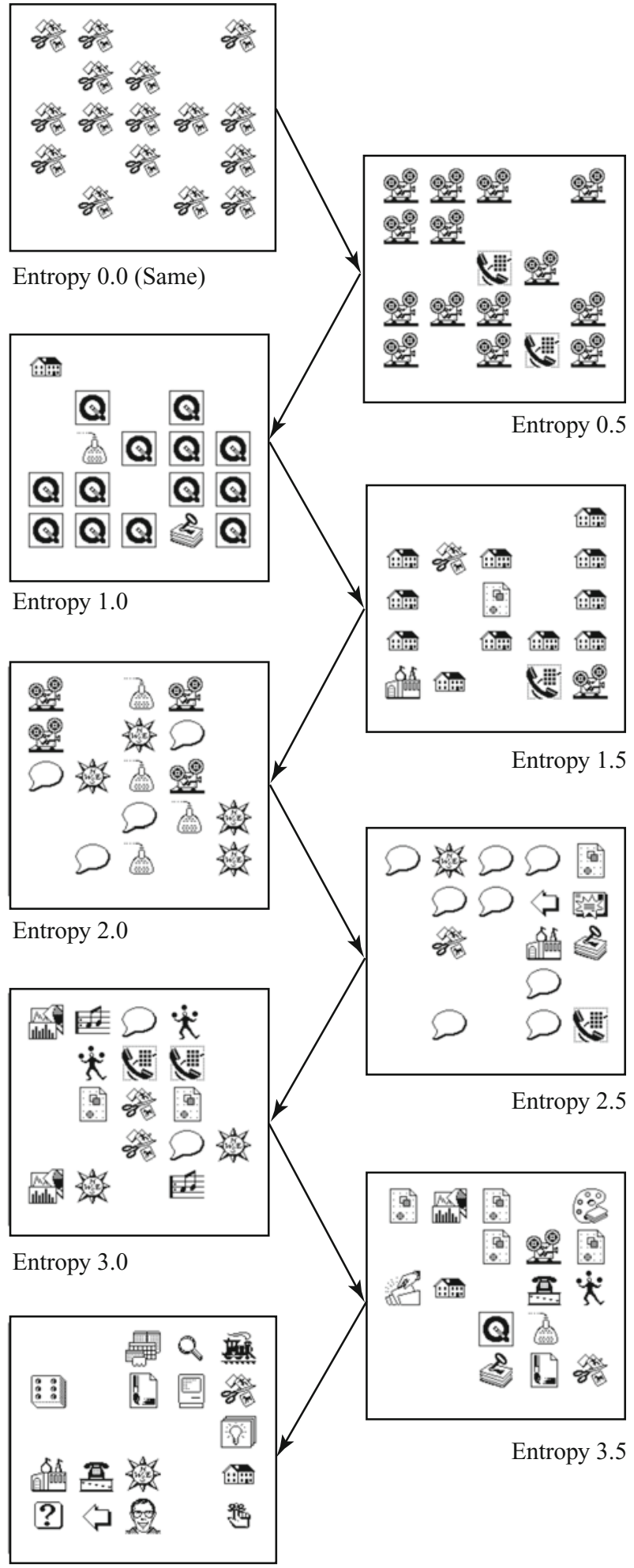

Entropy 4.0 (Different)

Figure 2. Examples of mixture arrays with different levels of entropy. 
nation of same arrays was relatively unaffected (Young, Wasserman, \& Garner, 1997, Experiment 1).

Importantly, this pattern of results can be explained if pigeons actually use display entropy to solve the discrimination. Better discriminative performance when 16-icon arrays are involved may be the result of the greater entropy disparity between 16-icon same and different arrays than between 2-icon same and different arrays. Computing entropy in these two situations reveals that the 2 -icon task requires distinguishing displays entailing entropies of 0.0 (same displays) and 1.0 (different displays), a relatively small disparity in entropy, whereas the 16-icon task requires distinguishing displays entailing entropies of 0.0 (same displays) and 4.0 (different displays), a much larger disparity in entropy (see Table 1).

Nonetheless, given that the pigeons in Young, Wasserman, and Garner (1997, Experiment 1) had first been trained with 16-icon arrays, it is possible that two factors may have influenced their responses to the smaller icon arrays; perhaps reducing the number of icons resulted in a stimulus generalization (novelty) decrement that was independent of the lowered entropy of the displays. In order to clarify the factors involved in the pigeons' performance, Young, Wasserman, and Garner (1997, Experiment 2) also trained other pigeons with arrays involving different number of icons (2-, 4-, 8-, 12-, or 16-icon same and different arrays) from the outset of the experiment. Given the ear- lier reported difficulties in obtaining strong same-different discrimination with two-item displays (e.g., Edwards et al., 1983; Santiago \& Wright, 1984; Wright et al., 1985; Wright et al., 1983), Young, Wasserman, and Garner hoped that concurrent training with larger number arrays would facilitate discrimination of the smaller number arrays. However, the pigeons did not learn to discriminate same from different arrays when only 2 or 4 icons were shown. Moreover, the smaller number arrays actually retarded learning of the larger number arrays. Here, too, discriminative performance to same arrays was largely unaffected by decreasing the number of icons, whereas discriminative performance to different arrays was detrimentally affected by decreasing the number of icons.

In a parallel comparative project, Wasserman, Fagot, and Young (2001) found that baboons quickly learned to discriminate same from different stimulus arrays comprising 16 icons. As well, the baboons reliably generalized the same-different discrimination to arrays of novel icons. Moreover, the baboons responded much like the pigeons to systematic changes in mixtures of same and different icons, suggesting that baboons, too, used the amount of visual display variability in their discrimination behavior. Finally, when the number of icons was decreased from the training value of 16, baboons' discrimination of same from different displays also fell dramatically (Wasserman, Young, \& Fagot, 2001).

Table 1

Statistical Attributes of the Same, Different, and Mixture Arrays

\begin{tabular}{|c|c|c|c|}
\hline $\begin{array}{l}\text { Type of } \\
\text { Array }\end{array}$ & Icon Arrangement & Entropy & $\begin{array}{l}\text { Number of } \\
\text { Icon Types }\end{array}$ \\
\hline Same & $\begin{array}{l}2 \text { icons } \\
4 \text { icons } \\
8 \text { icons } \\
12 \text { icons } \\
16 \text { icons }\end{array}$ & $\begin{array}{l}0.0 \\
0.0 \\
0.0 \\
0.0 \\
0.0\end{array}$ & $\begin{array}{l} \\
1 \\
1 \\
1 \\
1 \\
1\end{array}$ \\
\hline Different & $\begin{array}{l}2 \text { icons } \\
4 \text { icons } \\
8 \text { icons } \\
12 \text { icons } \\
16 \text { icons }\end{array}$ & $\begin{array}{l}1.0 \\
2.0 \\
3.0 \\
3.6 \\
4.0\end{array}$ & $\begin{array}{r}2 \\
4 \\
8 \\
12 \\
16\end{array}$ \\
\hline Same & $16 \mathrm{~A}$ & 0.0 & 1 \\
\hline Mixture & $\begin{array}{l}\text { 14A2B } \\
\text { *8A8B } \\
\text { *12A3B1C } \\
\text { "13A1B1C1D } \\
\text { 11A1B1C1D1E1F } \\
\text { 4A4B4C4D } \\
\text { 8A1B1C1D1E1F1G1H1I } \\
\text { "2A2B2C2D2E2F2G2H } \\
\text { "5A3B1C1D1E1F1G1H1IJ } \\
\text { "6A1B1C1D1E1F1G1H1I1J1K } \\
\text { 4A1B1C1D1E1F1G1H1IJ1K1L1M }\end{array}$ & $\begin{array}{l}0.5 \\
1.0 \\
1.0 \\
1.0 \\
1.5 \\
2.0 \\
2.5 \\
3.0 \\
3.0 \\
3.0 \\
3.5\end{array}$ & $\begin{array}{r}2 \\
2 \\
3 \\
4 \\
6 \\
4 \\
9 \\
8 \\
10 \\
11 \\
13\end{array}$ \\
\hline Different & 1A1B1C1D1E1F1G1H1I1J1K1L1M1N1O1P & 4.0 & 16 \\
\hline
\end{tabular}

Note-In the top half of the table are the same and different training arrays. In the bottom half of the table are the mixture testing arrays, which always comprised 16 icons, along with the 16-icon same and different training arrays, for comparison purposes. Each letter designates a randomly chosen icon type that was different from those designated by the other letters in a string. The numeral preceding a letter indicates the number of times the icon type occurred. *Mixture arrays with identical entropy levels (1.0 or 3.0) but created with a different number of icon types. 


\section{Entropy and Human Discrimination Behavior}

What about human beings? What strategy might they use to solve a nonverbal same-different discrimination? How might people respond to mixtures of some same and some different items or to arrays with all identical items (same arrays) and all nonidentical items (different arrays) that contain different numbers of visual items?

Here, we suspected that pigeons' and baboons' use of visual display variability to solve the same-different discrimination task might not occur in human beings. So, we conducted a parallel project with college students (Young \& Wasserman, 2001a). Human participants were told to observe a series of displays and to learn which of two arbitrary responses was correct for each of the displays. The participants did not actually report "same" and "different" to the visual displays but merely pressed one of two different keys; the words same and different were never used in the instructions, nor were the participants provided with any information that could have directed them toward any particular feature of the displays. The codes "same" and "different" are used in our article for purely expository purposes.

Using this paradigm, we did indeed find that most people $(80 \%)$ responded categorically to the testing arrays, predominately reporting "different" to all mixtures of some identical and some nonidentical items. But a minority (20\%) of our college student participants responded continuously to the mixture displays: As the displays gradually changed from low to high levels of variability, the participants' responses progressively changed from "same" to "different." As well, the latter participants exhibited the same peculiar pattern of reporting "same" to arrays of two or four nonidentical items that our pigeons had evidenced. This finding is uniquely explained by the decrease in variability or entropy that occurs when fewer nonidentical items are given; no such change in variability or entropy occurs when fewer identical items are given. Could it be that human behavior, too, is controlled by entropy?

Our pigeons (Young, Wasserman, \& Garner, 1997) and baboons (Wasserman, Young, \& Fagot, 2001) had previously evidenced worsening discrimination performance as the number of icons was reduced from 16 to 12 to 8 to 4 to 2. Although not so dramatically, our humans, too, showed a fall in choice accuracy, as well as a rise in reaction time (RT), as the number of icons was reduced (Young \& Wasserman, 2001a). But in the Young and Wasserman (2001a) project, participants had been trained with 16-icon arrays. The decrease in choice accuracy and the increase in RTs might not be due to the decrease in the number of icons; it might, instead, be due to the change in the number of icons from the training value, a stimulus generalization decrement. Because the testing displays containing fewer numbers of icons had never been seen before, performance might deteriorate due to a novelty effect that made it more difficult for the participants to apply what they had learned during the training phase. If this stimulus generalization interpretation were true, training people with $2,4,8,12$, and 16 icons from the outset should eliminate any effect of the number of items, since all of these numbers would be equally familiar. But if training with same and different displays of diverse numbers of icons still produced poor performance with displays of small numbers of icons, this result would attest to real discrimination difficulty when the number of items is small.

So, in the present study, we pursued humans' discrimination of multiple-item arrays by again training participants to make discriminative responses to identical and nonidentical arrays. Here, however, the training arrays did not always involve 16 items, as had been the case in our earlier studies; instead, the training arrays randomly involved 2, 4, 8, 12, or 16 items. When pigeons were trained with arrays of different numbers of icons from the outset of the experiment, they failed to learn to discriminate same from different arrays that involved only 2 or 4 icons, and they more poorly discriminated arrays with a larger number of icons (Young, Wasserman, \& Garner, 1997, Experiment 2). We did not expect the random number task to be arduous for human participants. But if the entropies of the displays do affect participants' performance, the present training could be considerably more difficult than that in which only 16-icon arrays are involved. Whereas the 16-icon task requires distinguishing between entropies of 0.0 (same displays) and 4.0 (different displays), as the number of icons falls, the disparity between same and different displays becomes progressively smaller; so, when only 2 icons are involved, the participants must distinguish between displays with entropies of 0.0 and 1.0 (see Table 1), a relatively small disparity that participants might find difficult.

In our experimental setting, displays with smaller numbers of icons also possessed an intrinsic property that might have influenced the participants' performance. The 16 icons in same or different displays were randomly distributed over 25 locations in a $5 \times 5$ grid, thereby leaving 9 locations blank. As can be seen in Figure 3, the mean distance between icons was greater with displays involving fewer icons than it was with displays involving more icons; perhaps this greater interitem distance is the reason why the number of icons affected discriminative performance.

Young, Wasserman, and Garner (1997) performed post hoc analyses for all possible two-icon displays to see whether their pigeons' accuracy would improve when the icons were close together; they found no significant effect of this interitem distance on the pigeons' discriminative performance. In order to experimentally explore this matter, we included two experimental conditions: In one, the icons were always distributed throughout the display, just as in our previous experiments (distributed condition); in the second, the icons appeared at the center of the screen, always immediately adjacent to one another, vertically, horizontally, or diagonally (grouped condition).

Unlike in our pigeon and baboon experiments, in the present investigation, we also recorded and analyzed the participants' RTs in making their discriminative report responses. Young and Wasserman (2001a) had earlier found that all of their research participants - even those who categorically discriminated the visual arrays - responded more quickly with increasing numbers of items in the displays. Again, 

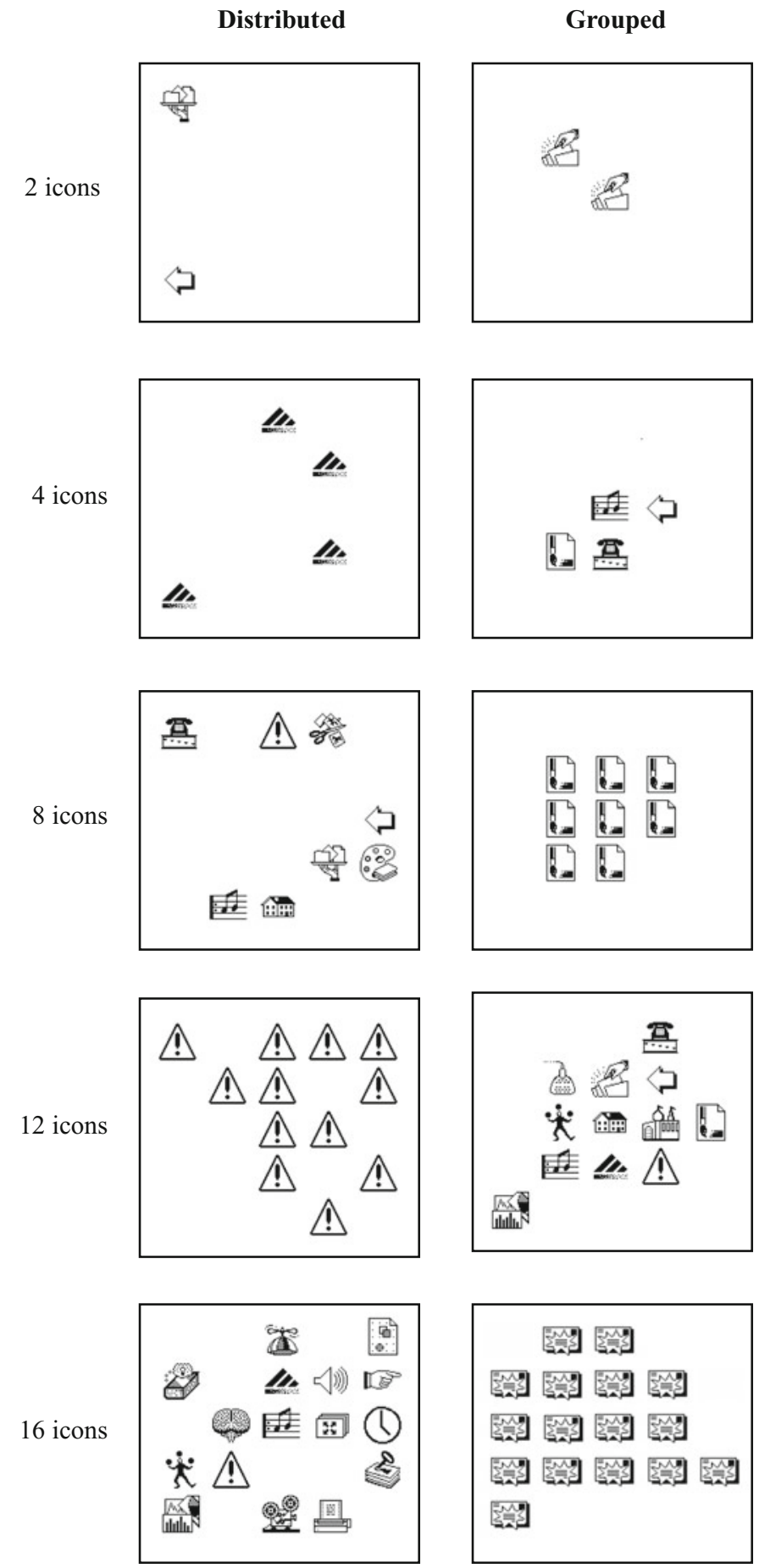

Figure 3. Examples of same and different 2-, 4-, 8-, 12-, and 16-icon arrays used in the present study. On the left are arrays with the icons randomly distributed; on the right are arrays with the icons grouped in the center.

because training in the present experiment was carried out with randomly presented arrays containing different numbers of items, any RT effects would be free of confounding by a stimulus generalization decrement. Moreover, if the fall in accuracy and the rise in RT with fewer icons observed by Young and Wasserman (2001a) were due to the greater distance between the icons, these effects should be replicated in the distributed condition, but not in the grouped condi- 
tion. On the contrary, both conditions should evidence these response trends if the number of icons is the critical factor in discriminative performance.

\section{METHOD}

\section{Participants}

A total of 250 introductory psychology students at the University of Iowa received course credit for their voluntary participation. The participants were randomly assigned to the distributed condition $(n=171)$ and to the grouped condition $(n=79)$.

\section{Visual Stimuli and Experimental Design}

The particular displays that the participants were presented on each of the trials comprised several icons chosen from a set of 24 icons. For any given same display, a single icon from the set was randomly chosen and was used to make up displays of $2,4,8,12$, or 16 icons. For any given different display, 2, 4, 8, 12, or 16 icons from the set were selected with no repetitions (see Figure 3 for examples of these displays).

In the distributed condition, we used our standard methodology of random icon distribution, in which icons are randomly distributed over 25 locations arrayed in a $5 \times 5$ grid, thereby leaving 9 or more locations blank. In the grouped condition, we used a new method of icon placement in which the icons were always immediately adjacent to one another (vertically, horizontally, or diagonally). This new method used the central matrix position on all displays. To create displays of 2, 4, or 8 icons, 1, 3, or 7 additional icons, respectively, were randomly located in the remaining 8 positions of the central $3 \times 3$ area. To create displays of 12 or 16 icons, the central $3 \times 3$ area was filled with 9 icons; 3 or 7 additional icons, respectively, were randomly located in the remaining 16 positions of the $5 \times 5$ grid.

\section{Procedure}

Training. The participants were seated at one of four identically configured 233-MHz iMac computers. The participants first heard general instructions and then read more specific instructions that explained the mechanics of the experiment and that exhorted the participants to make their choices as quickly as possible while still being accurate. These instructions also contained the following introductory paragraph:

You will be observing a series of displays and attempting to learn which response is correct for each display. You will make your best guess as to which of two responses is correct. You will then be provided feedback in the form of an auditory tone (if correct) or a flash of the screen (if incorrect). This information will assist you in improving your chances of being correct for subsequent displays. Your goal is to accurately predict the correct response for each display by the end of training.

No information was provided that could have directed the participant toward any particular aspect of the displays. At no point in the instructions were the words same or different used. Once each participant had indicated an understanding of the procedure, the experimenter started all of the programs for that contingent of participants.

The training period comprised 80 trials: 4 randomized blocks of 20 arrays each, 2 each of 2-, 4-, 8-, 12-, and 16-icon same and different arrays. For half of the participants, the "1" key was correct for same arrays and the " 3 " key was correct for different arrays; for the other half of the participants, the key assignments were reversed. The presentation of stimuli and the processing of responses were controlled by Hypercard, Version 2.3.

Each trial began with illumination of the display area with a white field containing a black cross in the center. The participant initiated a trial by pressing the " 2 " key, which turned on a black-on-white icon array. The participant then made a choice response ("1" or "3" key). A correct choice response was followed by a pleasant tone and the blackening of the display area. An incorrect choice response was followed by a flash of the display area (it rapidly changed from black-on-white to white-on-black and back again); the participant was not allowed to continue until the correct response had been made. These correction trials were not scored in data analyses. An illegal response (any response other than a " 1 " or " 3 ") was followed by an unpleasant tone. Intertrial intervals averaged $3 \mathrm{sec}$ (range: 2-4 sec).

Testing. After the 80 training trials, the testing period began. The session continued without a noticeable change, but the testing arrays were randomly interspersed among the training arrays at a relatively low rate. During testing, five randomized blocks of 51 trials were given, consisting of 40 same and different training arrays (4 each of 2-, 4-, 8-, 12-, and 16-icon arrays) and 11 different mixture arrays, one each with entropy levels of $0.5,1.5,2.0,2.5$, and 3.5 and three each with entropy levels of 1.0 and 3.0. The mixture arrays always comprised 16 icons. The particular mixtures that were used for producing these entropy scores is shown in Table 1. Figure 2 depicts some examples of these mixture displays.

We used three different methods for generating the Entropy 1.0 and Entropy 3.0 16-icon arrays as a manipulation check to ensure control by entropy, rather than by other properties of the displays (e.g., the number of icon types; see Young \& Wasserman, 2001a). In Method 1, each type of icon appeared an equal number of times, so that when entropy was 1.0 , there were 2 icon types presented 8 times each, and when entropy was 3.0 , there were 8 icon types presented twice each. In Method 2, one of the types of icons appeared 3 times, one other was allowed to appear more than once (12 times when entropy was 1.0 and 5 times when entropy was 3.0), whereas each of the others could appear only once. And in Method 3, one of the types of icons was allowed to appear more than once, whereas each of the others could appear only once, so that when entropy was 1.0 , there was one icon type presented 13 times and 3 icon types presented just once each, and when entropy was 3.0, one icon type was presented 6 times and 10 icon types were presented just once each.

Thus, the last 255 trials of a session consisted of 200 training trials with same and different arrays plus 55 mixture testing trials. Differential feedback continued for trials involving same and different arrays, so that a pleasant sound was presented if the response was correct and a flashing screen was presented, followed by repetition of the trial, if the response was incorrect. For mixture testing arrays, there were no correct or incorrect responses; "correct" feedback was always given, so that the participants would always hear the pleasant sound and would advance to the next trial regardless of their responses. In this way, repeated testing could be conducted without teaching the participants any particular response to these mixture arrays.

\section{RESULTS}

We chose an inclusion criterion of $70 \%$ correct on same and different trials across the entire experiment; any participant failing to meet this criterion was dropped from subsequent analyses. We adopted this criterion in order to obtain the clearest view of discriminative performance during testing. Of the 250 participants, 154 met this criterion; we retained 102 out of $171(60 \%)$ participants in the distributed condition and 52 out of $79(66 \%)$ participants in the grouped condition. ${ }^{1}$ All the subsequent analyses excluded the 80-trial training period.

\section{Choice Accuracy}

Figure 4 (left) illustrates that during the testing period, the participants in both the grouped and the distributed conditions accurately discriminated same from different 

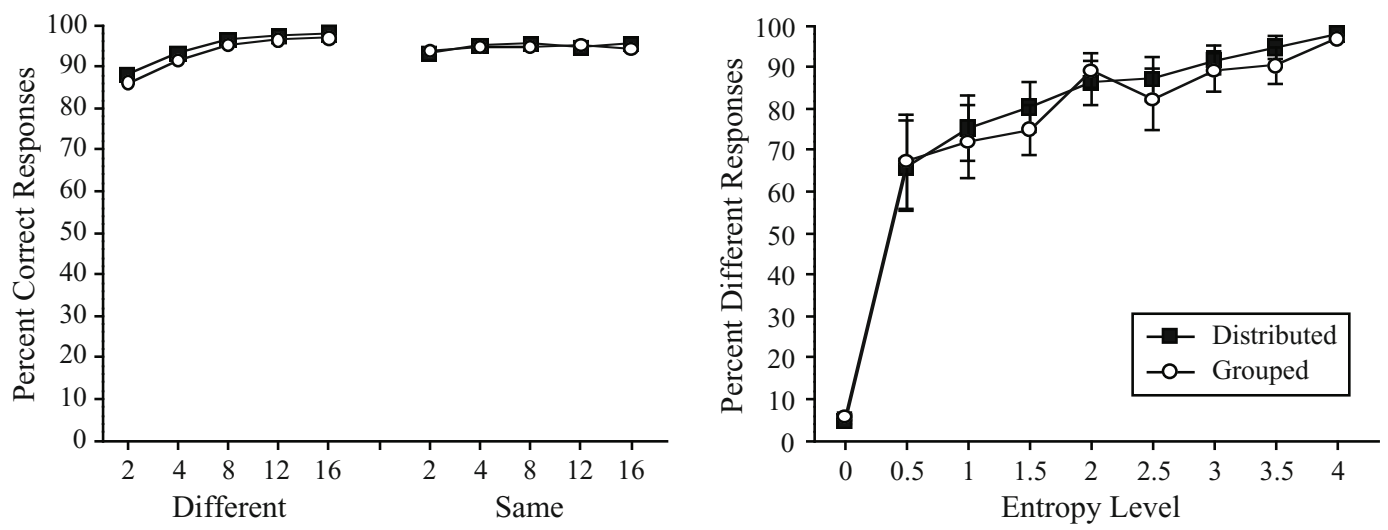

Figure 4. Left panel: Mean percentage of correct responses to the same and different arrays with different number of icons for the distributed and grouped conditions. Right panel: Mean percentage of "different" responses to the 16-icon displays with different levels of entropy for the distributed and grouped conditions. Error bars indicate the standard errors of the means.

arrays regardless of the number of icons. There was a tendency for the participants to perform more accurately when the number of icons was increased in different arrays, but not in same arrays. Figure 4 (right) illustrates that during the testing period, the participants' responding to the testing arrays comprising mixtures of same and different icons generally yielded reports of "different," with such reports rising as a function of display variability or entropy. At all nonzero levels of entropy, the participants made a preponderance of "different" reports.

More careful scrutiny of the participants' responding to the testing arrays disclosed that there were substantial individual differences in discriminative performance (cf. Young \& Wasserman, 2001a). To segregate the disparate response profiles, we performed Ward's (1963) hierarchical cluster analysis on the participants' percentage of "different" responses to the mixture arrays in each of the conditions and focused on the highest level two-cluster division in the dendrogram. In the distributed condition,
70 participants were in one cluster, and 32 were in the other; in the grouped condition, 36 participants were in one cluster, and 16 were in the other.

Figure 5 reveals that there were two substantially different patterns of responding to the mixture displays; these two patterns were equally represented in the grouped and the distributed conditions. The participants in the smaller clusters (distributed continuous, $n=32$, and grouped continuous, $n=16$ ) exhibited strong sensitivity to the full range of display variability in the mixture arrays (Figure 5, right); as the mixture was changed from mostly same to mostly different icons, these participants' responding changed from mostly "same" reports to mostly "different" reports. Thus, the participants in the continuous clusters responded as if display variability was measured along a continuous dimension. Interestingly, as can be observed in Figure 5 (left), these participants also evidenced a notable rise in accuracy to the different arrays as the number of items was increased, increasing from $75 \%$ for 2 -icon ar-
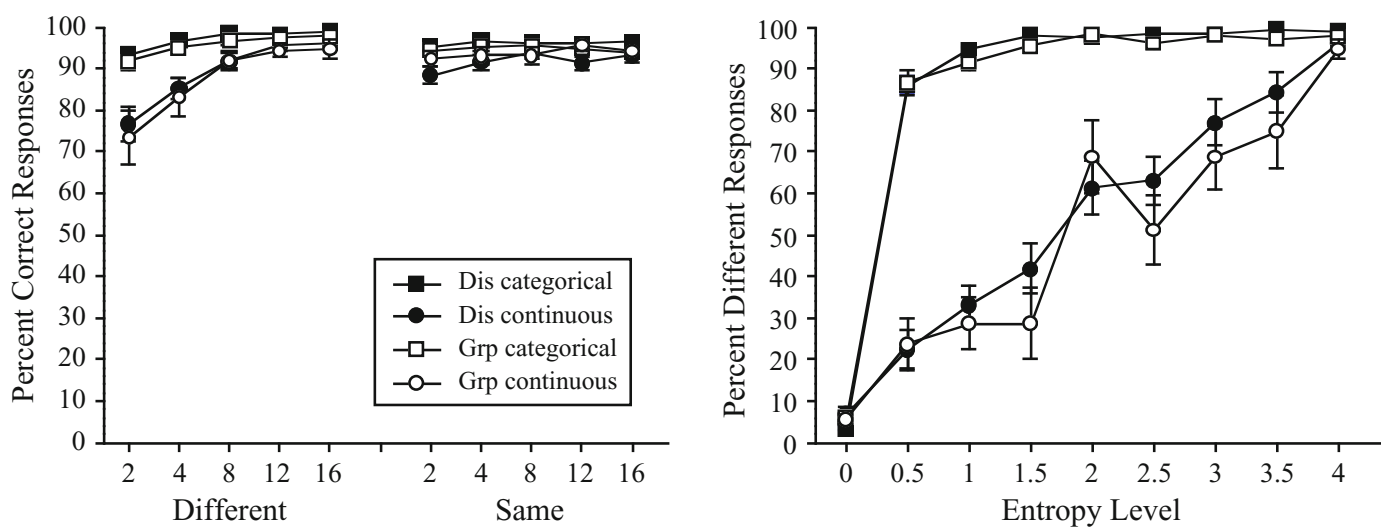

Figure 5. Left panel: Mean percentage of correct responses to the same and different arrays with different number of icons for the distributed (Dis) and grouped (Grp) conditions. Right panel: Mean percentage of "different" responses to the 16-icon displays with different levels of entropy for the distributed and grouped conditions. The response patterns are separately portrayed for the categorical and continuous participant clusters. Error bars indicate the standard errors of the means. 
rays to $95 \%$ for 16 -icon arrays (averaged across the distributed and the grouped conditions); icon number had a much smaller effect on their responding to the same arrays.

The participants in the larger clusters (distributed categorical, $n=70$, and grouped categorical, $n=36$ ) behaved dramatically differently, treating the discrimination categorically. When any of the icons were different, these participants tended to report "different"; only when all of the icons were identical did they consistently report "same" (Figure 5, right). There was some slight evidence that even these participants were sensitive to display variability, as revealed by their increasing tendency to choose the different key when entropy on mixture trials was increased. These participants were largely unaffected by the number of icons in the same and different displays, although they did exhibit a small increase in accuracy to the different arrays as the number of items was increased, rising from $93.5 \%$ for 2 -icon arrays to $98.0 \%$ for 16 -icon arrays (averaged across the distributed and the grouped conditions; see Figure 5, left).

To confirm these observations, we first examined the participants' performance to the same and different arrays. A significance level of .05 was chosen for this and all the subsequent tests. A $2 \times 2 \times 2 \times 5$ repeated measures mixed ANOVA of the percentage of "different" responses as a function of icon location (distributed or grouped), cluster (categorical or continuous), array type (same or different), and number of icons $(2,4,8,12$, or 16) revealed a significant main effect of cluster $[F(1,150)=$ 23.6, $\left.M S_{\mathrm{e}}=0.009\right]$, a significant main effect of array type $\left[F(1,150)=7,038.8, M S_{\mathrm{e}}=0.03\right]$, and a significant main effect of number of icons $\left[F(4,600)=31.7, M S_{\mathrm{e}}=\right.$ 0.004]. Also statistically significant were the cluster $\times$ array type interaction $\left[F(1,150)=27.9, M S_{\mathrm{e}}=0.031\right]$, the cluster $\times$ number of icons interaction $[F(4,600)=$ $\left.9.4, M S_{\mathrm{e}}=0.004\right]$, and the array type $\times$ number of icons interaction $\left[F(4,600)=66.5, M S_{\mathrm{e}}=0.003\right]$. There was no significant main effect of icon location, nor was this factor involved in any significant interaction. Most important, the three-way cluster $\times$ array type $\times$ number of icons interaction was significant $[F(4,600)=24.25$, $\left.M S_{\mathrm{e}}=0.003\right]$, as evidenced by the differential effects of icon number on same and different trials for the two clusters of participants (Figure 5, left).

Linear trend analyses disclosed that the continuous participants' accuracy rose as the number of icons was increased in different arrays $\left[F(1,150)=82.06, M S_{\mathrm{e}}=\right.$ $0.013]$; their accuracy also rose in same arrays as the number of icons was increased $\left[F(1,150)=8.27, M S_{\mathrm{e}}=\right.$ $0.003]$. Linear trend analyses divulged that the categorical participants' accuracy also rose as the number of icons was increased in different arrays $[F(1,150)=12.47$, $\left.M S_{\mathrm{e}}=0.013\right]$; their accuracy did not significantly change in same arrays $(F<1)$.

In a separate analysis, we considered the participants' performance for the mixture testing arrays. A $2 \times 2 \times 9$ repeated measures mixed ANOVA of percentage of "different" responses as a function of icon location (distrib- uted or grouped), cluster (categorical or continuous), and entropy level $(0.0,0.5,1.0,1.5,2.0,2.5,3.0,3.5$, or 4.0$)$ revealed a significant main effect of cluster $[F(1,150)=$ 282.32, $\left.M S_{\mathrm{e}}=0.108\right]$ and a significant main effect of entropy level $\left[F(8,1200)=477.39, M S_{\mathrm{e}}=0.018\right]$. There was also a significant icon location $\times$ entropy level interaction $\left[F(8,1200)=2.6, M S_{\mathrm{e}}=0.018\right]$, indicating that the percentage of "different" responses was slightly lower in the grouped condition as the entropy level increased (Figure 5, right). Most important, the significant cluster $\times$ entropy level interaction $\left[F(8,1200)=99.18, M S_{\mathrm{e}}=0.018\right]$ underscored that the two clusters of participants were decidedly different in their treatment of the arrays with different levels of display entropy, regardless of the grouped or distributed presentation of the icons.

Finally, we analyzed the mixture displays with entropy levels of 1.0 and 3.0. These displays had been created using different methods, so that we had three different types of Entropy 1.0 and Entropy 3.0 displays, but composed from different numbers and frequencies of icon types (see Table 1). If the continuous participants' responses were controlled by entropy and no other properties of the displays, there should be no differences among the three different types of displays sharing the same entropy level. On the contrary, any differences found among these displays would challenge the entropy explanation. The categorical participants' performance to these displays is theoretically less interesting; they should not show any differences either, simply because entropies greater than 0 (when at least one icon is different from the rest of the icons) ought to prompt "different" reports, regardless of the variability of the displays.

For Entropy 1.0 displays, the continuous participants' "different" responses did not vary significantly when the displays comprised 2,3 , and 4 icon types $[F(2,94)=1.71$, $\left.M S_{\mathrm{e}}=0.044\right]$. For Entropy 3.0 displays, the continuous participants" "different" responses did not vary significantly either $\left[F(2,94)=1.15, M S_{\mathrm{e}}=0.029\right]$. This lack of significant differences supports entropy as the dimension controlling the continuous participants' performance. The categorical participants' "different" responses were also very similar for each of these sets of displays. For Entropy 1.0 displays, $F(2,210)=0.75, M S_{\mathrm{e}}=0.006$, and for Entropy 3.0 displays, $F(2,210)=0.49, M S_{\mathrm{e}}=0.001$.

Therefore, when the number of icon types was varied but entropy was held constant, the participants' responses did not significantly vary. The categorical participants' responses were "different" in all cases, regardless of the level of entropy, whereas the continuous participants" "different" responses were low for Entropy 1.0 displays and high for Entropy 3.0 displays and were very similar within each of these sets of displays. Therefore, the continuous participants' responses are genuinely affected by entropy, and not by other characteristics of the displays, such as the number of icon types or the relative frequency of a particular icon.

\section{Reaction Time}

We next examined the participants' RTs to same and different arrays that comprised different numbers of icons 
and to the mixture arrays. Figure 6 (left panel) shows that the participants' RTs in the distributed condition were very similar to the participants' RTs in the grouped condition. In both conditions, the participants' $\mathrm{RT}^{2}{ }^{2}$ were shorter to same arrays $(M=668$ and $641 \mathrm{msec}$ for the distributed and the grouped conditions, respectively) than to different arrays ( $M=736$ and $738 \mathrm{msec}$ for the distributed and the grouped conditions, respectively). As well, RTs progressively fell as the number of icons in the displays was increased for both same and different arrays. When the mixture arrays contained different levels of entropy (Figure 6, right), the participants' RTs in the distributed and the grouped conditions were also very $\operatorname{similar}(M=$ 761 and $800 \mathrm{msec}$, respectively). In each case, RTs rapidly rose from Entropy 0.0 (same arrays) to Entropy 0.5 displays, followed by a steady fall in RTs as entropy declined from 0.5 to 4.0 (different arrays).

More detailed scrutiny of the behavior of the different clusters of participants disclosed quite disparate patterns of performance. First, examining performance to the same and different displays of icons (Figure 7, left), we observed that the participants' RTs were very similar for same arrays with different numbers of icons; RTs generally fell with increases in the number of icons. For different arrays, however, the categorical participants (in both the distributed and the grouped conditions) executed shorter RTs than did the continuous participants $(M=$ 640 and $953 \mathrm{msec}$, respectively); this disparity increased with increasing numbers of icons in the display.

A difference between the categorical and the continuous participants was also observed in arrays entailing different levels of entropy, with the former executing shorter RTs than did the latter $(M=683$ and $998 \mathrm{msec}$, respectively). This RT disparity was seen only when the displays involved entropies equal to or greater than 1.0 (Figure 7, right).

To confirm the reliability of these RT observations, we first examined the participants' RTs to same and different testing arrays. A $2 \times 2 \times 2 \times 5$ repeated measures mixed ANOVA of mean $\log (\mathrm{RT})^{3}$ (the logarithmic transform was used to normalize the RTs) as a function of icon location (distributed or grouped), cluster (categorical or continuous), array type (same or different), and number of icons $(2,4,8,12$, or 16) revealed a significant main effect of cluster $\left[F(1,150)=8.5, M S_{\mathrm{e}}=0.087\right]$, a significant main effect of array type $\left[F(1,150)=29.05, M S_{\mathrm{e}}=0.014\right]$, and a significant main effect of number of icons $[F(4,600)=$ $\left.49.4, M S_{\mathrm{e}}=0.001\right]$. Also statistically significant were the cluster $\times$ array type interaction $[F(1,150)=54.86$, $\left.M S_{\mathrm{e}}=0.014\right]$, the icon location $\times$ number of icons interaction $\left[F(4,600)=3.14, M S_{\mathrm{e}}=0.001\right]$, and the cluster $\times$ number of icons interaction $\left[F(4,600)=5.94, M S_{\mathrm{e}}=\right.$ $0.001]$.

Most important, the significant three-way cluster $\times$ array type $\times$ number of icons interaction $[F(4,600)=$ $\left.17.07, M S_{\mathrm{e}}=0.0002\right]$ confirmed the differential effects of icon number in same and different displays for the two clusters of participants (Figure 7, left). In same displays, RTs were similar for the participants in all the conditions, showing a common pattern of slightly decreasing RT as the number of icons was increased. This pattern was confirmed by linear trend analyses $\left[F(1,150)=51.52, M S_{\mathrm{e}}=\right.$ 0.009 , and $F(1,150)=23.42, M S_{\mathrm{e}}=0.009$, for the distributed continuous and the grouped continuous participants, respectively; $F(1,150)=36.13, M S_{\mathrm{e}}=0.009$, and $F(1,150)=7.48, M S_{\mathrm{e}}=0.009$, for the distributed categorical and the grouped categorical participants, respectively]. In different displays, on the other hand, RTs were less uniform. The categorical participants in both the distributed and the grouped conditions showed marked drops in RT as the number of icons was increased. This pattern was confirmed by linear trend analyses $[F(1,150)=60.25$, $M S_{\mathrm{e}}=0.008$, and $F(1,150)=19.82, M S_{\mathrm{e}}=0.008$, for the distributed categorical and the grouped categorical participants, respectively]. The continuous participants' RTs, although similar to the categorical participants' RTs when the displays contained two icons, showed only a slight decline when the number of icons was increased for the distributed continuous participants [linear trend analysis fell just short of statistical significance; $F(1,150)=3.41$,
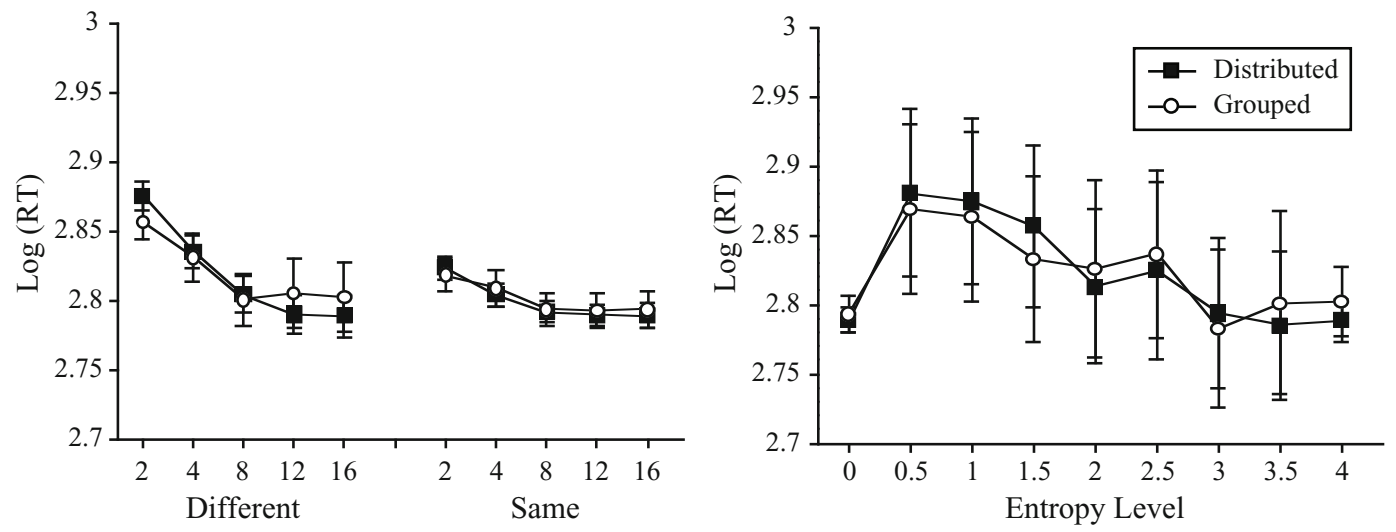

Figure 6. Left panel: Mean reaction times (RTs) to the same and different arrays with different numbers of icons for the distributed and the grouped conditions. Right panel: Mean RTs to the 16-icon displays with different levels of entropy for the distributed and the grouped conditions. Error bars indicate the standard errors of the means. 

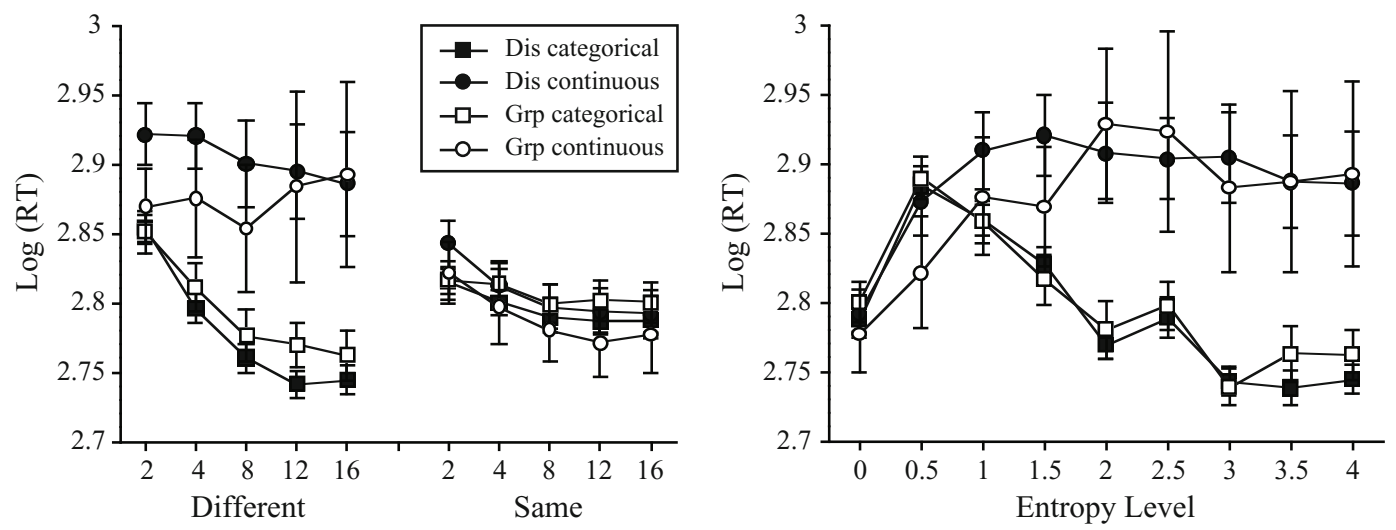

Figure 7. Left panel: Mean reaction times (RTs) to the same and different arrays with different numbers of icons for the distributed and the grouped conditions. Right panel: Mean RTs to the 16-icon displays with different levels of entropy for the distributed (Dis) and the grouped (Grp) conditions. The RTs are separately portrayed for the categorical and continuous participant clusters. Error bars indicate the standard errors of the means.

$\left.M S_{\mathrm{e}}=0.008, p=.06\right]$, whereas there was no reduction at all for the grouped continuous participants $[F(1,150)=$ $\left.0.55, M S_{\mathrm{e}}=0.008\right]$.

We next considered the participants' RTs to the mixture testing arrays. A $2 \times 2 \times 9$ repeated measures ANOVA of $\log (\mathrm{RT})$ as a function of icon location (distributed or grouped), cluster (categorical or continuous), and entropy level $(0.0,0.5,1.0,1.5,2.0,2.5,3.0,3.5$, or 4.0$)$ revealed a significant main effect of cluster $[F(1,150)=$ $\left.15.47, M S_{\mathrm{e}}=0.116\right]$, a significant main effect of entropy level $\left[F(8,1200)=26.32, M S_{\mathrm{e}}=0.004\right]$, and a significant cluster $\times$ entropy level interaction $[F(8,1200)=31.05$, $\left.M S_{\mathrm{e}}=0.004\right]$. Increases in entropy led to an abrupt rise and fall in RT for the categorical participants, the function peaking at 0.5 ; increases in entropy led to a more gradual rise in RT for the continuous participants, with only a slight fall beyond 2.0.

\section{DISCUSSION}

Even when the participants had been trained from the outset with displays containing different numbers of icons, we observed a general improvement in same-different discrimination performance as the number of icons in the display was increased. On same trials, accuracy was very high and largely unaffected by the number of items (Figure 4, left); RTs were short, but even they shortened as the number of items was increased (Figure 6, left). On the other hand, accuracy was lower and RTs were longer on different trials, with both accuracy rising (Figure 4, left) and RTs becoming considerably shorter (Figure 6, left) as the number of items was increased.

Performance on mixture trials revealed two strikingly disparate patterns of discrimination behavior. As in our previous study (Young \& Wasserman, 2001a), people's predominant inclination ( $70 \%$ of our participants) was to treat the visual displays categorically, with the remainder ( $30 \%$ of our participants) responding continuously to visual display variability. The participants in the categorical cluster responded "different" when any of the icons were different; only when all of the icons were identical did they report "same" (Figure 5, right). Accordingly, their RTs were short with Entropy 0.0 (same) arrays; they rose sharply with Entropy 0.5 arrays (where the category boundary between same and different presumably lies), and they became progressively shorter as they approached the opposite categorical endpoint with the Entropy 4.0 (different) arrays (Figure 7, right). Hence, the categorical participants' RTs to the mixture arrays disclosed that they had the greatest difficulty discriminating displays with entropies near the category boundary. Prior studies involving physical dimensions such as size or length have also shown that RTs increase as response uncertainty increases near categorical boundaries (Ashby, Boynton, \& Lee, 1994). By contrast, the continuous participants responded as if display variability fell along a continuous dimension: As the mixture displays were changed from including mostly the same icons to mostly different icons, these participants' responding gracefully changed from mostly "same" reports to mostly "different" reports (Figure 5, right). The continuous participants' RTs to these mixture arrays rose from Entropy 0.0 to Entropy 2.0, followed by a slight fall to Entropy 4.0, suggesting that the categorical boundary between low and high variability was less distinct in this group and that it was located near Entropy 2.0 (Figure 7, right).

Hence, we clearly documented that both different numbers of icons and different levels of visual display entropy importantly influence people's same-different discrimination behavior. However, we found no effect of the spatial distribution of the icons in a display. In previous studies, the icons were randomly located in the $5 \times 5$ array; therefore, the average distance between icons was greater for displays involving fewer icons. This greater distance might have increased the participants' difficulty in processing displays containing small numbers of icons. In the pres- 
ent study, the icons were randomly located for some participants (distributed condition) and were clustered in the central part of the display for other participants (grouped condition). Regardless of the distributed or grouped location of the icons in the display, the participants again exhibited more errors and longer RTs with reduced numbers of icons. Therefore, our previously reported data (Young $\&$ Wasserman, 2001a) are unlikely to represent mere generalization decrement. There truly seems to be an intrinsic difficulty for people to discriminate same from different stimulus arrays involving small numbers of items.

Prior research has shown that decreasing the numbers of icons in visual arrays has a large detrimental impact on other species' discrimination of same from different displays (pigeons, Young, Wasserman, \& Garner, 1997; baboons, Wasserman, Young, \& Fagot, 2001). This effect of the number of items on same-different discrimination performance can be readily explained by entropy. When the number of items is small (e.g., 2 icons), the task requires distinguishing displays with an entropy of 0.0 (same displays) from displays with an entropy of 1.0 (different displays), whereas when the number of items is large (e.g., 16 icons), the task requires distinguishing entropies of 0.0 and 4.0, a much greater disparity that ought to make the 16 -icon task much easier. Furthermore, changes in the number of items ought to have a much greater effect on responding to different arrays than to same arrays; entropy systematically increases on the former trials but remains constant at 0.0 on the latter trials.

Nonetheless, entropy alone cannot explain all of our results. As we just noted, when visual displays contain identical icons (same displays), entropy is always the same, 0.0, regardless of the number of icons. Yet, even with same displays, we observed that RTs grew longer when the displays contained fewer icons for both continuous and categorical participants. Accuracy too decreased slightly to same displays containing fewer icons for the continuous participants. These performance decrements to same displays had also been observed by Young and Wasserman (2001a). But in our earlier study, these decrements could be attributed to a generalization decrement from the 16-item training displays; the participation of generalization decrement was eliminated in the present study.

\section{Other Accounts}

Similarity. Young and Wasserman (2001a) did consider other possible bases for people's discriminating the variability of visual arrays. Continuous participants might have responded on the basis of the pairwise similarity of items in the array. Young and Wasserman (2001a) constructed a pairwise similarity measure, which was scored by computing the number of pairwise matches among each of the items in a given array; they used pairwise matches, rather than pairwise mismatches, to create the observed asymmetry in performance where reducing the number of items had an effect on different arrays, but not on same arrays. The resulting pairwise similarity scores always pro- duced fits that were inferior to the entropy measures; thus, it was not further considered (see Young \& Wasserman, 2001a, for a detailed analysis).

Coactivation. Research with the redundant-target paradigm has revealed a performance advantage when information is provided by a larger number of items (e.g., Miller, 1982; Mordkoff \& Yantis, 1991; van der Heijden, La Heij, \& Boer, 1983). In a redundant-target task, performance on trials in which only one target is given is compared with that on trials in which two or more identical targets are given. For example, participants may have to detect the presence of a target letter, X. An RT advantage is normally observed when a trial contains two Xs, relative to when a trial contains only a single X (cf. Kinchla, 1974). According to coactivation models (e.g., Miller, 1982), responses to redundant signals are especially fast because two or more sources provide additional activation to trigger the appropriate response. Activation naturally grows faster when it is provided by several sources, rather than by only one. In the case of our same and different displays - where all of the relationships among the icons in a given display are of sameness or differentness - we might say that as the number of items increases, the number of relations of sameness or differentness also increases. In a 2-icon display, there is only one relationship involving those two individual icons; but in a 16-icon display, multiple relationships are present for each of the icons with the other icons. The larger the number of relationships, the more sources of activation there are for the appropriate response, yielding better performance-lower RTs and higher accuracies - as the number of items increases.

Predictions for mixture trials, in which some of the items are the same as one another and some of the items are different from one another, are more difficult to make. Presumably, same activation would compete with different activation, with the relationship more widely represented activating a response. This mechanism would not explain the categorical participants' behavior (because with just one item different, they chose "different" even when all the rest were the same), but it could fit the continuous participants' gradual response pattern on mixture trials. Still, if simply similarity to the rest of the items determines the participant's response, the coactivation account would reduce to pairwise similarity, which has been shown to be inferior to the entropy account (Young \& Wasserman, 2001a). Also, coactivation models would not predict any difference in responding to same and different displays (we observed a much larger effect of the number of items on different trials) or the different patterns of discrimination behavior shown by the categorical and the continuous participants.

Finding differences model. A given array can be understood as a collection of items each of which can be active to a certain degree. It can be further stated that items that differ from their neighbors receive greater activation than do those that are similar to their neighbors and that these different levels of activation can be combined into an activation map (e.g., Wolfe, 1994). Young, Ellefson, 
and Wasserman (2003) outlined a variability discrimination model in which each item in the display has a certain activation value. The activation of an item is a function of, among other factors, the degree of difference between it and each of the adjacent items. When a display involves high variability, the display comprises many items that are different from their neighbors, so the map contains many highly active items. On the other side, in a display involving low variability, there are many items that are very similar to one another, so the map contains few active items. The combination of these different levels of activation results in an activation map portraying different levels of variability. The activation map thus represents a computational redescription of the display. After a display was redescribed as an activation map, Young et al. (2003) averaged across the activation values to produce an aggregate measure of the differences that were present in the array. This average activation was mapped to a discriminative response through the reinforcement contingencies of the task.

The finding differences model can explain the influence of variability on the behavior of pigeons, baboons, and our continuous participants: As display variability increases, the different key is more likely to be chosen, because each item's activation is increased (it is more likely to differ from its neighbors). As well, the model predicts that discriminative performance should worsen as the number of items decreases, but only on different displays (the average activation of a same display is always 0.0 ). The model accounts for the behavior of our categorical participants through the use of its response rule (Luce's decision rule); the boundary between categories is assumed to have shifted and been sharpened, due to previous learning experiences.

\section{DIFFERENT PATTERNS OF BEHAVIOR}

Although mechanisms in addition to entropy might participate in same-different discrimination behavior, the similar effects of different numbers of items and levels of variability in several species strengthen our suspicion that variability discrimination lies at the root of same-different discrimination performance. Nonetheless, although humans' same-different discrimination behavior appears to be strongly influenced by entropy, humans' discriminative performance differs from that of pigeons and baboons in a fundamental way: After training with the endpoints (same and different) of the variability continuum, most people are inclined to treat the discrimination of intermediate degrees of variability in a categorical fashion (see also Young \& Wasserman, 2001a), whereas all pigeons (Young \& Wasserman, 1997) and all baboons (Wasserman, Fagot, $\&$ Young, 2001) treat the discrimination in a continuous manner.

The possibility of qualitatively distinct learning systems has been raised by, among others, Ashby and Maddox (2005). Categorical learning could be based on rules, which are guided by an explicit reasoning process and are easy to describe verbally. On the other hand, categorical learning could be the result of information integration processes, in which information from two or more stimulus dimensions is integrated at some predecisional stage, so that the optimal strategy is difficult or impossible to describe verbally. According to Ashby and Maddox, these different learning mechanisms depend on the type of task that is being performed, although other authors argue that people can apply multiple procedures of categorization to the same task (e.g., Smith, Patalano, \& Jonides, 1998). In our study, task-related factors cannot have produced the different patterns of behavior, because the task was the same for all the participants. So, the question of why people exhibit different patterns of behavior still remains.

One interesting possibility is that humans' extensive language experience from very early ages leads people to reserve the verbal response "same" for a much narrower class of stimuli than the verbal response "different." Thus, the word same denotes a much more restrictive stimulus set than does the word different. Because humans, but not pigeons or baboons, can use these verbal responses as mediational devices in the present experimental procedures, humans' preponderant response profiles may differ markedly from those of nonhuman animals.

This linguistic interpretation might be tested in several ways. For example, we could try to reduce the involvement of humans' verbal behavior in our experimental procedures. We could test preverbal children. Or we could overload adults' verbal processing capabilities by requiring them to perform a demanding verbal task while they were concurrently engaged in the same-different discrimination. In either case, humans might then respond more like pigeons or baboons; then, such verbal mediation might explain the striking differences between animals' and most humans' same-different discrimination behavior.

These and other researchable ideas suggest that much more can and should be learned about variability discrimination in order to reach a fuller understanding of the mechanisms involved and the factors influencing this important behavioral capability.

\section{A FINAL NOTE ON NOMENCLATURE}

Here and in our prior report (Young \& Wasserman, 2001a), we segregated our human research participants into two clusters on the basis of their responding to different numbers of items and to different mixtures of same and different items. We termed those clusters continuous and categorical because of the highly distinctive patterns of performance that they exhibited.

We wish to be particularly clear that these cluster labels do not mean that altogether different processes actually governed the participants' discrimination behavior. In fact, we believe that entropy strongly controlled the behavior of both continuous and categorical participants.

The case for the continuous participants is clear. As entropy was increased on mixture trials, they progressively increased their reports of "different." And as the number 
of items was increased, the accuracy of their reports on both same trials and different trials increased, with RTs on same trials also falling. But the categorical participants also exhibited behavioral control by entropy. As entropy was increased on mixture trials beyond 0.0 , these participants progressively increased their reports of "different," although far less dramatically than did the continuous participants. And, as the number of items was increased, the accuracy of these participants' reports on different trials increased, with RTs falling on both same and different trials. So, if the behaviors of both categorical and continuous participants are affected by entropy, is there a real line separating these two patterns of responding? Perhaps not.

Goldstone and Barsalou (1998) have advanced the notion of a continuum from perceptual to conceptual representations, arguing that concepts usually stem from perception and that these perceptual origins never completely vanish. If the very root of the same-different concept involves the discrimination of variability, the evident signs of control by entropy in categorical responders can be readily understood. The distinction between categorical and continuous responders is, then, not itself a categorical one, but a matter of degree concerning how much impact entropy exerts. This creative proposal certainly merits careful future study.

\section{REFERENCES}

Ashby, F. G., Boynton, G., \& Lee, W. W. (1994). Categorization response time with multidimensional stimuli. Perception \& Psychophysics, 55, 11-27.

AsHBY, F. G., \& MADDOx, W. T. (2005). Human category learning. Annual Review of Psychology, 56, 149-178.

EDWARDS, C. A., JAGIELO, J. A., \& ZenTALl, T. R. (1983). "Same/different" symbol use by pigeons. Animal Learning \& Behavior, 11, 349-355.

GOLDSTONE, R. L., \& Barsalou, L. W. (1998). Reuniting perception and conception. Cognition, 65, 231-262.

Kaufman, E. L., LoRD, M. W., Reese, T. W., \& Volkmann, J. (1949). The discrimination of visual number. American Journal of Psychology, 62, 498-525.

KINCHLA, R. A. (1974). Detecting target elements in multielement arrays: A confusability model. Perception \& Psychophysics, 15, 149158.

Mackintosh, N. J. (2000). Abstraction and discrimination. In C. Heyes \& L. Huber (Eds.), The evolution of cognition (pp. 123-141). Cambridge, MA: MIT Press.

Miller, J. (1982). Divided attention: Evidence for coactivation with redundant signals. Cognitive Psychology, 14, 247-279.

MordKoff, J. T., \& YANTIS, S. (1991). An interactive race model of divided attention. Journal of Experimental Psychology: Human Perception \& Performance, 17, 520-538.

Santiago, H. C., \& Wright, A. A. (1984). Pigeon memory: Same/ different concept learning, serial probe recognition acquisition, and probe delay effects on the serial-position function. Journal of Experimental Psychology: Animal Behavior Processes, 10, 498-512.

SHANNON, C. E., \& WEAVER, W. (1949). The mathematical theory of communication. Urbana: University of Illinois Press.

Smith, E. E., Patalano, A. L., \& JonideS, J. (1998). Alternative strategies of categorization. Cognition, 65, 167-196.

TAVES, E. H. (1941). Two mechanisms for the perception of numerousness. Archives of Psychology, No. 265.

TRICK, L. M., \& PYLYSHYN, Z. W. (1994). Why are small and large numbers enumerated differently? A limited-capacity preattentive stage in vision. Psychological Review, 101, 80-102. van der Heijden, A. H. C., La Heis, W., \& Boer, J. P. A. (1983). Parallel processing of redundant targets in simple visual search tasks. Psychological Research, 45, 235-254.

WARD, J. H., JR. (1963). Hierarchical grouping to optimize an objective function. Journal of the American Statistical Association, 58, 236244.

WASSERman, E. A., Fagot, J., \& Young, M. E. (2001). Same-different conceptualization by baboons (Papio papio): The role of entropy. Journal of Comparative Psychology, 115, 42-52.

Wasserman, E. A., Hugart, J. A., \& KirKPatrick-Steger, K. (1995). Pigeons show same-different conceptualization after training with complex visual stimuli. Journal of Experimental Psychology: Animal Behavior Processes, 21, 248-252.

WASSERMan, E. A., YounG, M. E., \& CoOK, R. G. (2004). Variability discrimination in humans and animals: Implications for adaptive action. American Psychologist, 59, 879-890.

Wasserman, E. A., YounG, M. E., \& FAGOT, J. (2001). Effects of number of items on the baboon's discrimination of same from different visual displays. Animal Cognition, 4, 163-170.

Wolfe, J. M. (1994). Guided Search 2.0: A revised model of visual search. Psychonomic Bulletin \& Review, 1, 202-238.

Wright, A. A., Santiago, H. C., Sands, S. F., Kendrick, D. F., \& Cook, R. G. (1985). Memory processing of serial lists by pigeons, monkeys, and people. Science, 229, 287-289.

Wright, A. A., Santiago, H. C., Urcuioli, P. J., \& Sands, S. F. (1983). Monkey and pigeon acquisition of same/different concept using pictorial stimuli. In M. L. Commons, R. J. Herrnstein, \& A. R. Wagner (Eds.), Discrimination processes (Quantitative Analyses of Behavior, Vol. 4, pp. 295-317). Cambridge, MA: Ballinger.

Young, M. E., Ellefson, M. R., \& Wasserman, E. A. (2003). Toward a theory of variability discrimination: Finding differences. Behavioural Processes, 62, 145-155.

Young, M. E., \& WaSSERMan, E. A. (1997). Entropy detection by pigeons: Response to mixed visual displays after same-different discrimination training. Journal of Experimental Psychology: Animal Behavior Processes, 23, 157-170.

YoUNG, M. E., \& WASSERMAN, E. A. (2001a). Entropy and variability discrimination. Journal of Experimental Psychology: Learning, Memory, \& Cognition, 27, 278-293.

Young, M. E., \& Wasserman, E. A. (2001b). Evidence for a conceptual account of same-different discrimination learning in the pigeon. Psychonomic Bulletin \& Review, 8, 677-684.

Young, M. E., \& Wasserman, E. A. (2002). Detecting variety: What's so special about uniformity? Journal of Experimental Psychology: General, 131, 131-143.

Young, M. E., Wasserman, E. A., \& Dalrymple, R. M. (1997). Memory-based same-different conceptualization by pigeons. Psychonomic Bulletin \& Review, 4, 552-558.

Young, M. E., Wasserman, E. A., \& GARNER, K. L. (1997). Effects of number of items on the pigeon's discrimination of same from different visual displays. Journal of Experimental Psychology: Animal Behavior Processes, 23,491-501.

\section{NOTES}

1. Many participants failed to learned the task to criterion in the allotted time period. In Young and Wasserman (2001a), participants were trained to master the same task, but only with 16-icon same and different displays. In that study, only $7 \%$ of the participants did not reach the learning criterion ( $75 \%$ correct in the final training block). In the present study, this percentage rose to $38 \%$ (even when we used a slightly more lenient criterion to allow us to include a larger number of participants), but the present task was clearly more difficult. Here, the training displays comprised 2, 4, 8, 12, or 16 icons; however, the number of icons was an irrelevant factor, so that people had to learn to attend to one dimension of the displays (whether or not the icons were all the same or all different) and to ignore another highly salient factor (number of icons). Another possible explanation for this learning failure, perhaps more relevant theoretically, might be that the participants who did not learn were actually the ones who were most strongly controlled by entropy. As described in the text, when only 16-icon arrays are involved, there is 
a large entropy disparity between 16-icon same and different arrays, so that discrimination should be easy. As the number of icons is reduced, this disparity becomes progressively smaller, and consequently, the task should become harder. Dropped participants' accuracy on different trials was $M=46.3(S E=1.7), M=50.7(S E=1.5), M=55.6(S E=1.8)$, $M=58.3(S E=1.9)$, and $M=60.7(S E=2.1)$ for displays comprising $2,4,8,12$, and 16 icons, respectively. Dropped participants' accuracy on same trials was $M=58.4(S E=1.7), M=59.9(S E=1.6), M=59.9$ $(S E=1.8), M=60.1(S E=1.9)$, and $M=60.8(S E=1.8)$ for displays comprising $2,4,8,12$, and 16 icons, respectively. This pattern is just what would be expected if discrimination performance were influenced by entropy. However, the generally poor level of discriminative performance of these participants does not permit us to say anything decisive as to whether these participants were strongly affected by entropy or insufficiently attentive to their task.

2. Geometric means are used for all the RT data, as is recommended for highly skewed data.

3. The RTs in this and all the subsequent analyses included only correct trials to the training stimuli (these involved differential feedback) and all trials to the testing stimuli (these involved nondifferential feedback).

(Manuscript received March 28, 2005;

revision accepted for publication September 6, 2005.) 